\title{
Intelligent Mechatronic System for Automatically Evaluating the Training of the Laparoscopic Surgeon
}

\author{
Minor A. ${ }^{1}$, Lorias D. ${ }^{1}$, Ortiz Simon ${ }^{2}$ and Escamirosa F. ${ }^{1}$ \\ ${ }^{1}$ Centro de Investigación y de Estudios Avanzados del IPN \\ Instituto Tecnológico de Nuevo Laredo \\ México
}

\section{Introduction}

Laparoscopic surgery is seen as the surgery of the future. Its low mortality rate, its fast recuperation rate and the aesthetic advantages it offers the patient make it adequate for application in all of the medical sub-specialisms. This is the main reason why technological innovation for this surgery is accelerated. However, all this technology is insufficient if the specialist surgeon does not also use training and evaluation [Dankelman 2005, Martin et al. 1997, Vassiluo et al. 2005].

Training in the development of the laparoscopic surgeon continues to be the cornerstone of the specialism. It is for this reason that the surgeon should count with training methods that augment their skills and, even more than that, intelligent mechatronic systems that can measure this improvement in their skills. Current training systems are based principally on the time taken to complete tasks and the quality of the development of these tasks. [Peters et al. 2004, Chung \& Sackiier 1998, Keyser et al. 2000)].

Time is an objective and impartial factor, but measures of quality are normally subjective. Systems for training and for the evaluation of virtual reality, such as the haptic systems, [MaClusky et al., Sutherland et al. 2006], rate the quality of the training, but the cost of this evaluation is very high. For this reason cheaper mechatronic systems are required, whose philosophy of evaluation is based on the work developed by the user and the evolution of his learning.

In this way it could be possible to objectively evaluate the increase in the skill level achieved by the surgeon. The TrEndo system [Magdalena et al., 2006] is a good example of this effort to complement the measurement of quality of the training, but, even today, no methodology for establishing the clarity of this measurement has been developed. For this reason, we propose a new methodology of intelligent training evaluation, using the analysis in time of the three-dimensional behavior of each instrument during the execution of the task of transfer, based on a mechatronic system.

\section{Development}

In order to measure the evolution of training, a mechanotronic system was developed. This system carries out the three-dimensional registration of each instrument during the execution of each training task. 
Each laparoscopic training task has a particular objective. This could be visuo-spatial adaptation or the choreography of both hands, etc.

The design of the system that digitalizes the temporal position of each instrument during the execution of the task, in conjunction with the practice model, was selected taking three of the most commonly used environments for learning laparoscopic techniques into consideration [Keyser et al., 2000] (the virtual environment, direct practice in the operating theatre and the use of physical trainers). The operating theatre is the most realistic environment for evaluation. In this environment, the equipment and its interaction with the surroundings provide a natural feedback of the effort used in surgery. Unfortunately, it is not possible to carry out the evaluation of a novice surgeon in these conditions. These virtual and haptic systems are expensive and this substantially limits their practical application. The physical trainer is also a realistic and versatile environment [Schijven \& Jakimowicz, 2003], which, just like in the operating theatre, has a natural feedback through the use of the equipment. For this reason, a physical practice model was used, and a mechatronic system was designed which, in conjunction with a program of analysis developed in Matlab (the MathWorks, Inc.), evaluates the laparoscopic skills during the execution of a training task, see figure 1 .

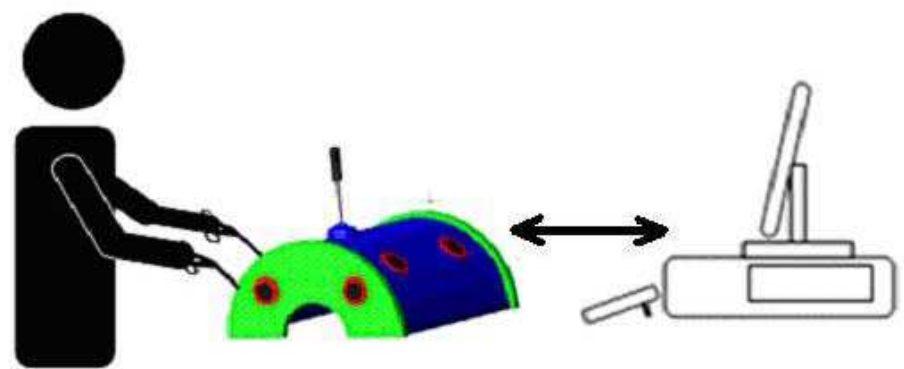

Fig. 1. Schematic diagram of the mechatronic laparoscopic evaluation system

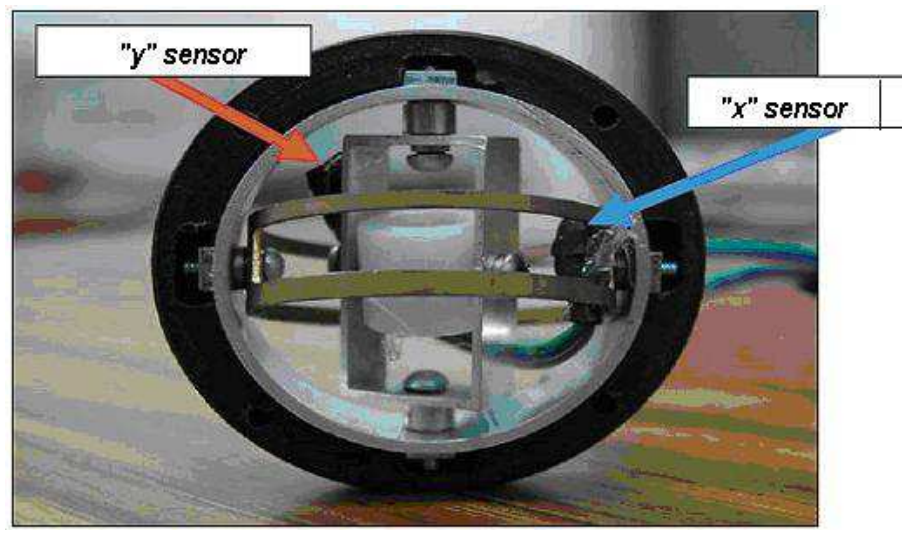

Fig. 2. Gimbal-type structure and assembly of the accelerometers for the registering movement on the $\mathrm{x}$ and $\mathrm{y}$ axes. 


\subsection{Three-dimensional registration system}

A mechatronic system of movement registration $(X, Y, Z)$ of the active type was designed. This consisted of a pair of accelerometer type sensors, (ADX330) which register the movement in the $\mathrm{X}$ y $\mathrm{Y}$ axes respectively. The reading of the movement in the $\mathrm{z}$ axis is measured using a resistive multiturn sensor. The sensors were joined with a gimbal-type structure and in a commercial trocar of $5 \mathrm{~mm}$ (see figures 2 and 3 )

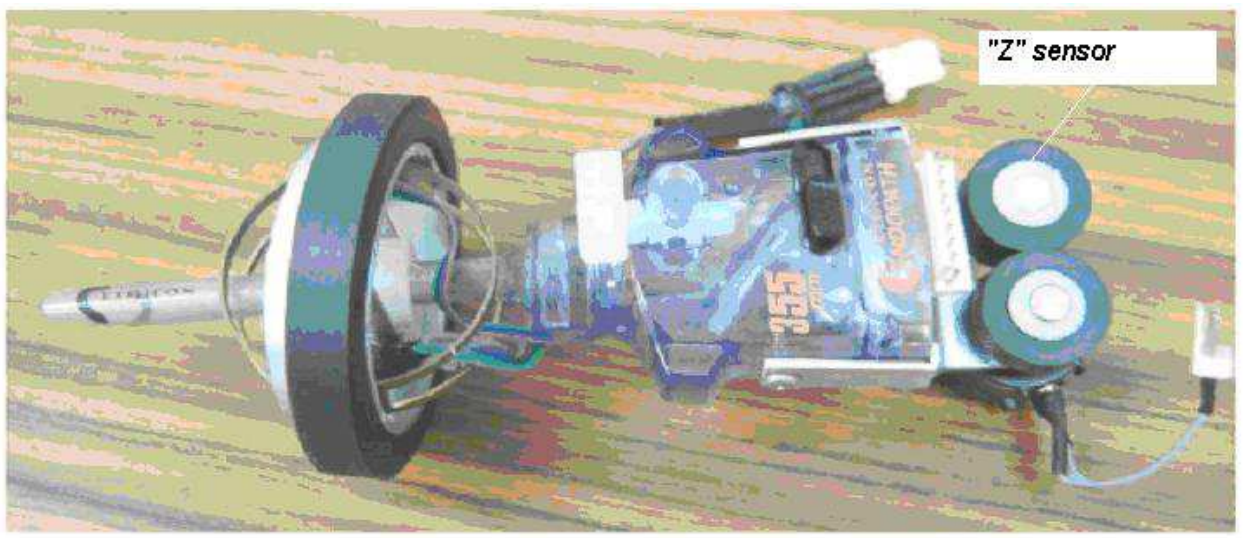

Fig. 3. Assembly of the trocar in the gimbal arrangement and location of the resistive sensor that measures the variable of movement along the $\mathrm{z}$ axis.

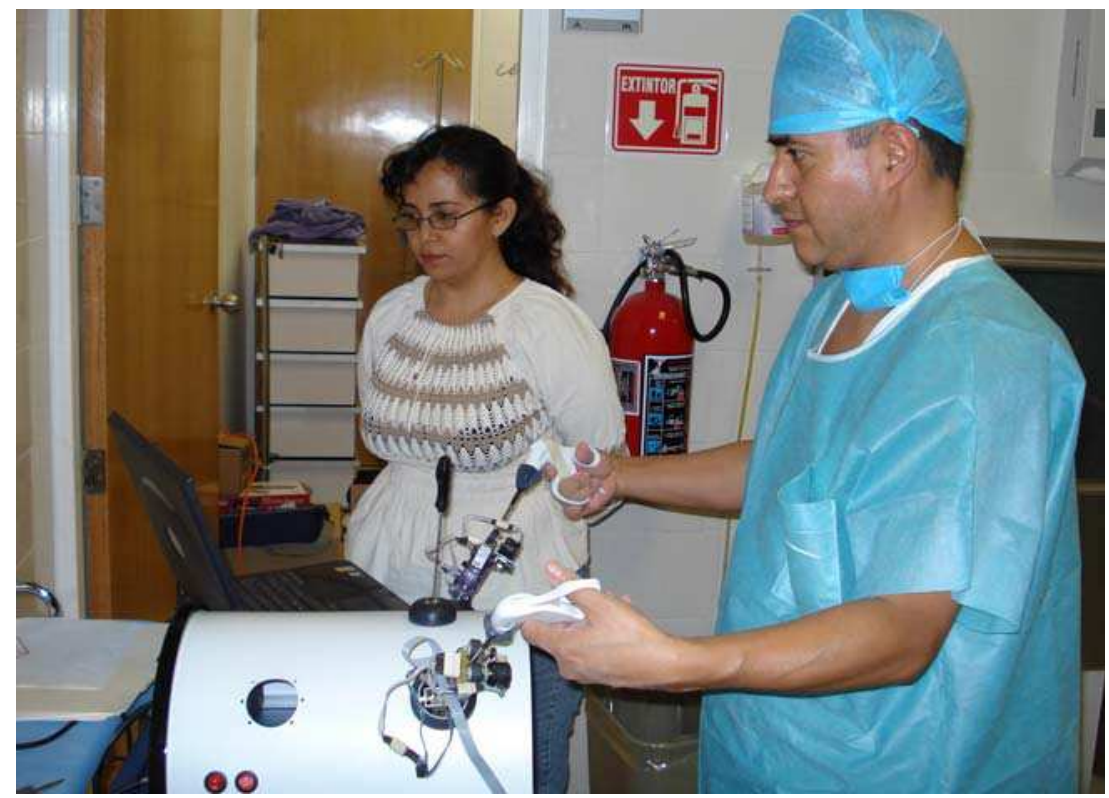

Fig. 4. General set up of the instrumented trochar installed in the trainer. The image shows the arrangement during the training. 
The main advantage of this arrangement is that equipment of any make or model can be used without the need for any costly modification of the equipment. In figure 4 the general 'fitting together' of the mechatronic unit can be seen; it is joined together with laparoscopic equipment.

Referring to the sampling frequency, the specifications of the protocol of communication allow a communication of up to $1 \mathrm{Mb} / \mathrm{s}$. Defining a channel of communication of 115.2 $\mathrm{kb} / \mathrm{s}$. And the frequency of sampling was adjusted via software to $16 \mathrm{~Hz}$.

Transfer of data

The information provided by the mechatronic system is sent to an electronic unit that conditions the digital data of 8 bits through a series of filters. These data are then sent to the computer through the USB port.

\subsection{User interface}

A program in the Matlab programming platform was designed that reads the data from the electronic unit. These data are processed in order to calculate different kinematic parameters

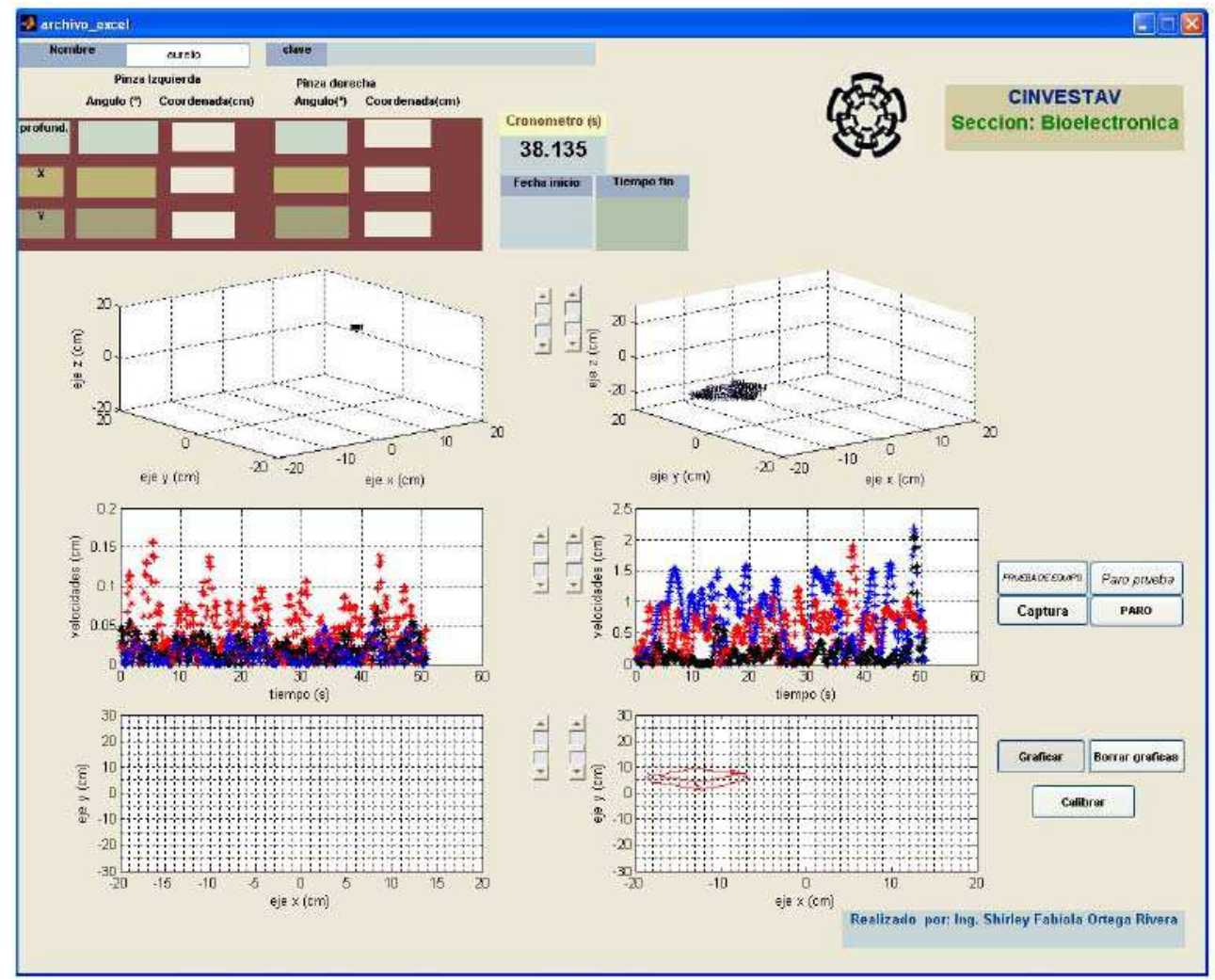

Fig. 5. Graphic interface of communication between the mechatronic system of movement digitalization and the user; Where the kinematic performance for both hands is shown in an independent and personalized form for each task. 
during the execution of the task such as: velocity $(\mathrm{cm} / \mathrm{S})$, movement $(\mathrm{cm})$, time $(\mathrm{s})$, spatial position of each forcep $\left(\mathrm{X}_{(t)}, \mathrm{Y}_{(t)}, \mathrm{Z}_{(t)}\right)$ for total time $(\mathrm{t})$. As well as the angles of inclination $(\phi)$. These data are processed independently and are presented for each hand, see fig. 5 .

\subsection{Calibration of the mechatronic evaluation system}

Calibration is very important and necessary for ensuring precision in the registration of laparoscopic movements. This calibration consists of adjusting the workspace variable, which is defined as the reach of the equipment. For carrying out this calibration the limits of inclination $(\phi)$ and the longitude of insertion (L) of the equipment are adjusted. See figure 6 . On executing the calibration command, this is carried out automatically. A series of messages appear that tell us the position in which the equipment should be placed. The total time for this routine lasts less than 2 seconds.

Once the calibration of the system is finished, it is possible to graph the trajectory of the laparoscopic equipment of each hand on carrying out a specific task in 3D.
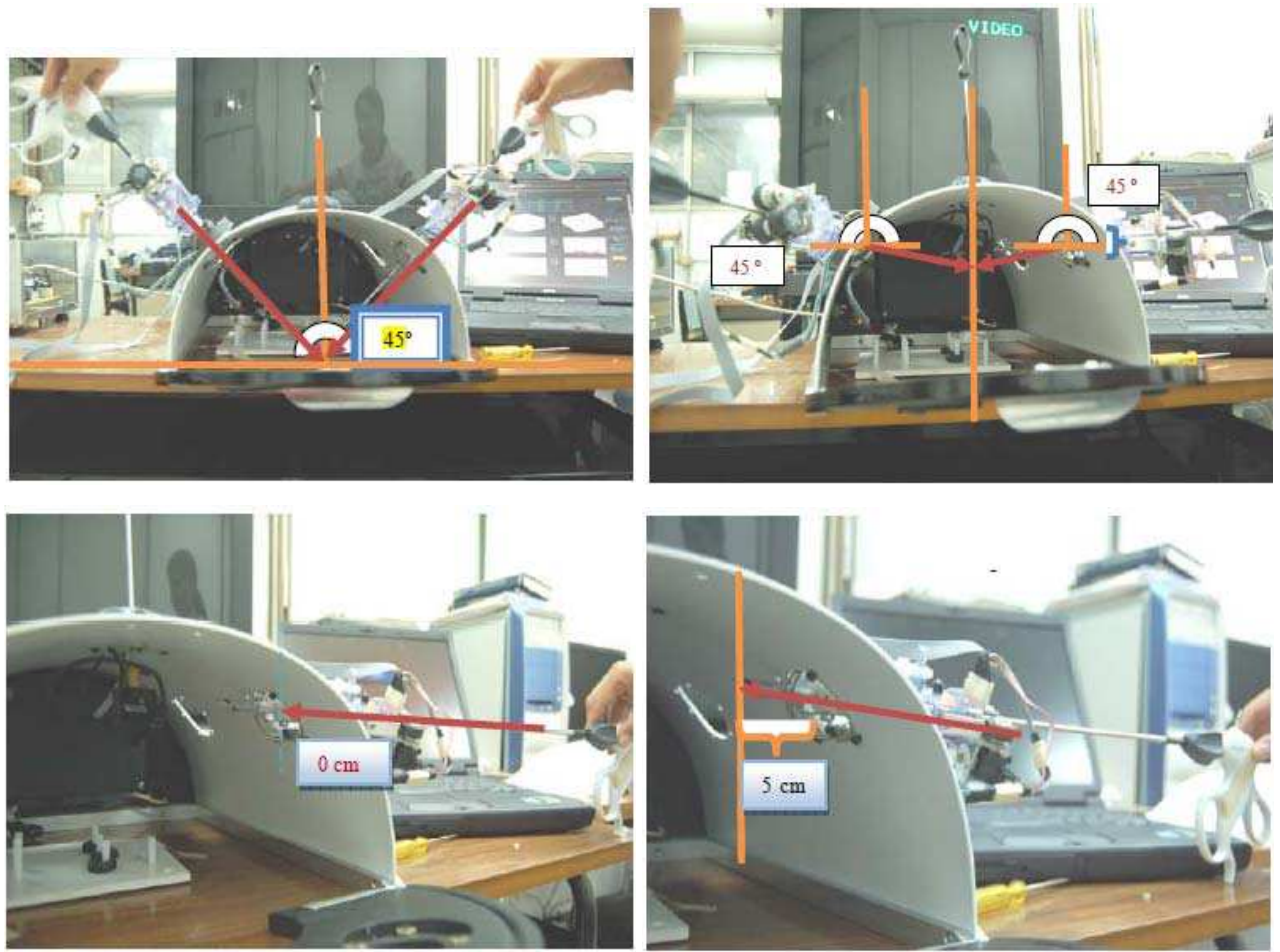

Fig. 6. Calibration of the system during which the workspace variable is adjusted, determined by the inclination $(\phi)$ and longitude of insertion (L) of the laparoscopic equipment.

The following figure 7 shows a three-dimensional graph of one task that consists of following the contour of a three-dimensional figure, which is placed inside the trainer, with the point of the laparoscopic instrument. 


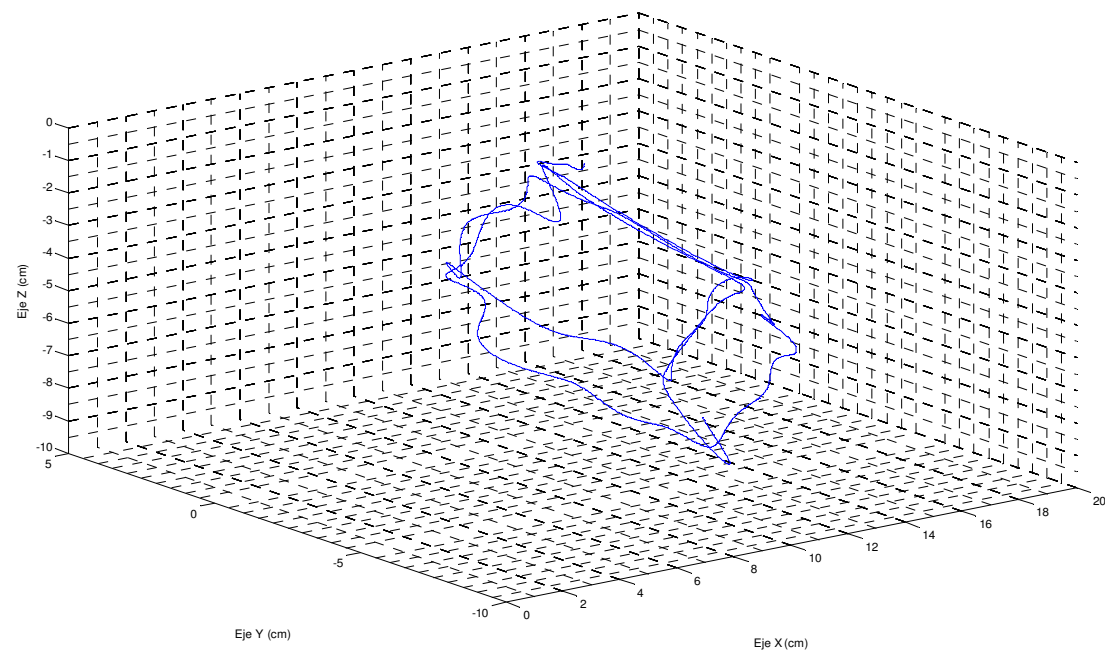

Fig. 7. Three-dimensional presentation of the $x, y, z$ registration of the mechatronic system once it is calibrated

\section{Automatic evaluation of laparoscopic training.}

The task selected to demonstrate automatic evaluation is the task of transfer of the McGill Inanimate System for Training and Evaluation of Laparoscopic Skills (MISTELS) protocols [Derossis et. al., 1998]. This task has two main objectives: to develop visuo-spatial adaptation and to develop synchronization between both hands in the training.

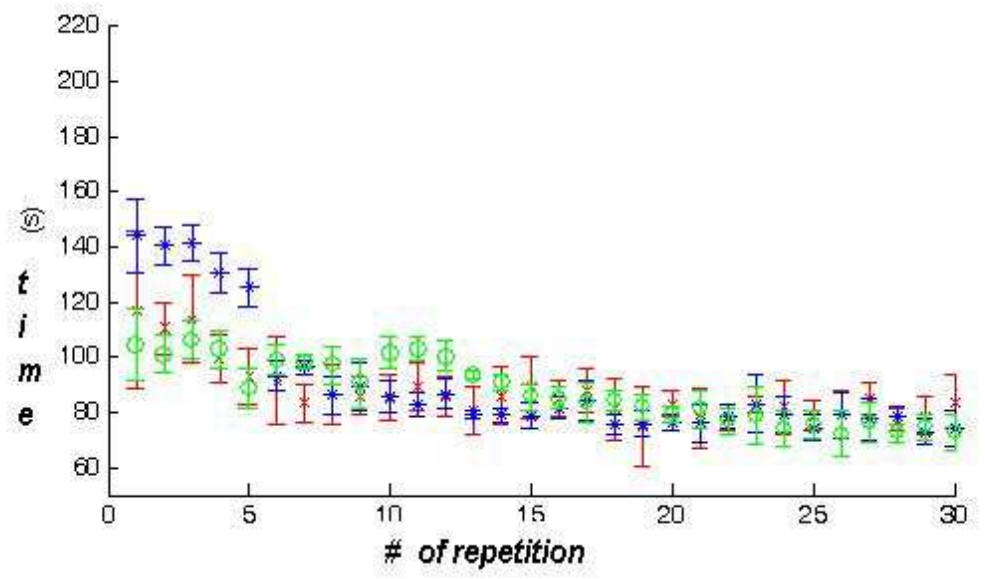

Fig. 8. A typical learning curve of one training task

This graph fig. 8 shows the natural evolution of learning (Grantcharov et al., 2003). As more repetitions of the same task are carried out, the time taken diminishes until it reaches one half, in this moment, the student has dominated the task. 
The Mistels transfer task consists of fig (9): The use of a plane with 12 poles, divided in two parts, 6 poles in each section. In one section, each pole is placed in a circle, for this reason it has a section which is full with 6 circles, these circles are transferred in the air from the left laparoscopic instrument to the right laparoscopic instrument, in the middle area of the plane.

Initially, they pass from the left to the right side, and the afterwards from right to left. Registers are taken in time and through this task visuo-spatial adaptation, and the choreography of both hands are evaluated.

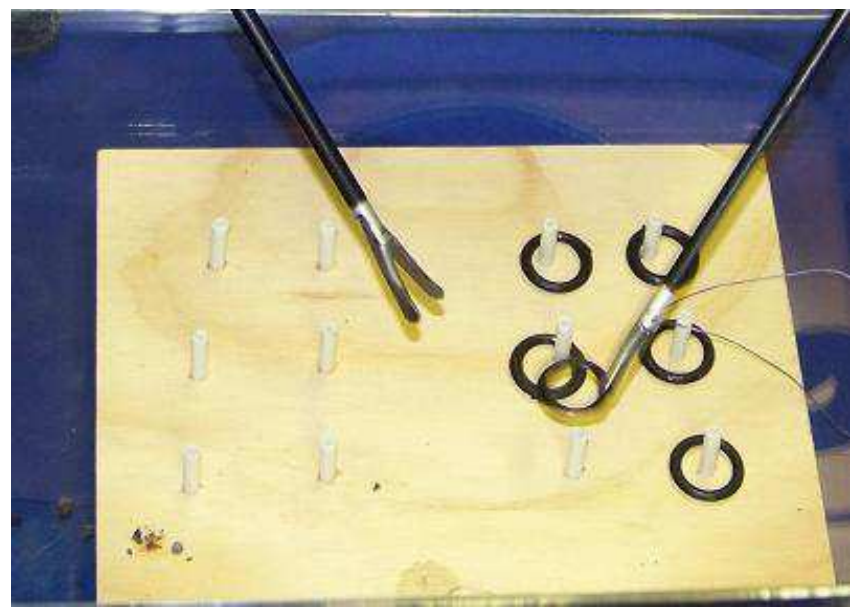

Fig. 9. Mistels Transfer task

The mechatronic system enables the registering of the temporal behavior of each instrument, permits the graphing of the dynamic behavior of each instrument in each plane fig. and produces a statistic evaluation of individual behavior in velocity, acceleration and the time taken to complete the task fig. 10.
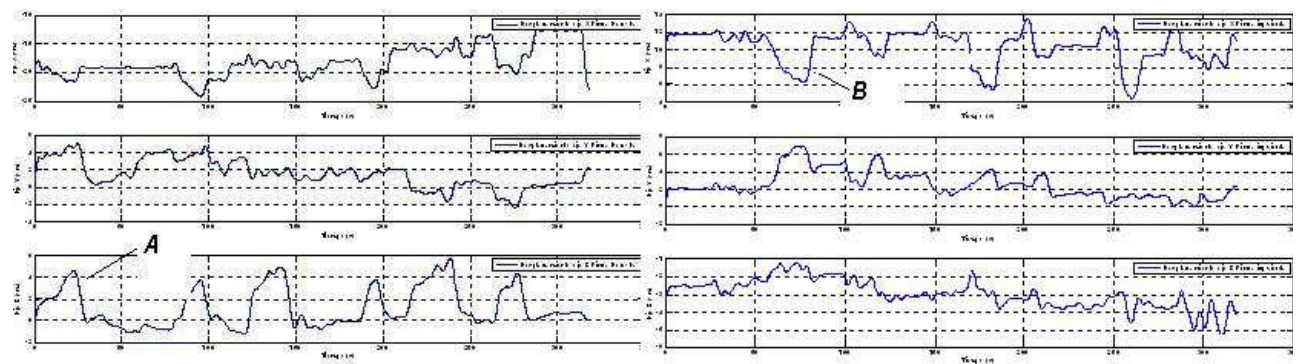

Right Forcep

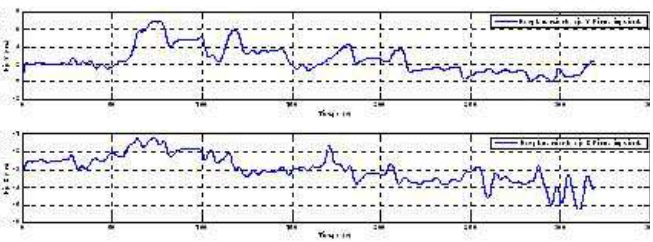

Left Forcep

Fig. 10. In some of these graphs, it is possible to clearly observe, when the circles are taken (A) with an instrument and afterwards, when they are deposited (B) with the other instrument.

From these graphs it is possible to calculate the low area of the curve. The low area of the curve represents the work, or effort, invested in carrying out the task. 


$$
\mathrm{W}=\int_{\mathrm{x}_{\mathrm{i}}}^{\mathrm{x}_{\mathrm{f}}} F_{\mathrm{x}}(\mathrm{x}) \mathrm{dx}
$$

We propose the calculation of the work (W), or effort, as an "intelligent" factor in evaluation of learning, as long as the work, or effort, invested is directly related with the development of the task:

The work in discreet form is:

$$
\mathrm{W} \approx \sum \mathrm{F}_{\mathrm{x}}(\tilde{\mathrm{x}}) \Delta \mathrm{x}
$$

Our hypothesis is: The resident expends less effort in carrying out a laparoscopic training task at the end of this. And if this effort can be calculated, then it is possible to use this value as an immediate objective factor that is capable of evaluating if a laparoscopic surgeon has dominated each laparoscopic training task.

If the task is carried out by a resident at the beginning of the training we have unwanted movements because of the lack of visuo-spatial adaptation, and the lack of hand-eye coordination. This is translated into the excessive time taken in developing the task and in abrupt movements during its execution.

If the same resident carries out the task after practicing the same task, he can complete the task in less time, and without such abrupt movements.

This value can be compared in any moment with itself or against a group. In this way, it is possible to evaluate the level of skill of each laparoscopic surgeon, whether the surgeon is novice, intermediate or experienced.

For the evaluation, 10 resident laparoscopes in the first year of their specialism were selected, without a close relationship with the handling of the laparoscopic equipment and with the dominant right hand. The transfer task of the Mistels protocol was selected, using optics of zero degrees.

\section{Results}

Data was registered and the average low area of the registration curve was calculated in the three planes $x, y$, and $z$, of the right forcep fig. 11 .

\section{Discussion}

A new automatic factor of evaluation of laparoscopic training was designed using the transfer task of the MISTELS protocol and a mechatronic system for the dynamic registration of the equipment.

To objectively measure the evolution of laparoscopic training is a technological challenge that, in being resolved, would permit the establishment of new parameters of immediate evaluation of the surgeon, whether he is novice, intermediate or expert. For this reason we propose the "work" as a new factor for evaluating laparoscopic training.

The results of the calculation of the "work" at the beginning of training, demonstrate that the surgeon invests greater energy in both hands. When visuo-spatial adaptation and synchronization between both hands are better, the effort invested starts to diminish substantially, until it reaches the average value.

According to the results, before the training of the surgeon uses more energy on not having enough ability to adapt himself visually, and through coordination, to the resolution of the 
task. On the other hand, on developing the skill, the surgeon invests less work in developing the same task in less time. One important characteristic is that this parameter can be calculated automatically from the registered data and can give an objective parameter of evaluation of the training for each hand.

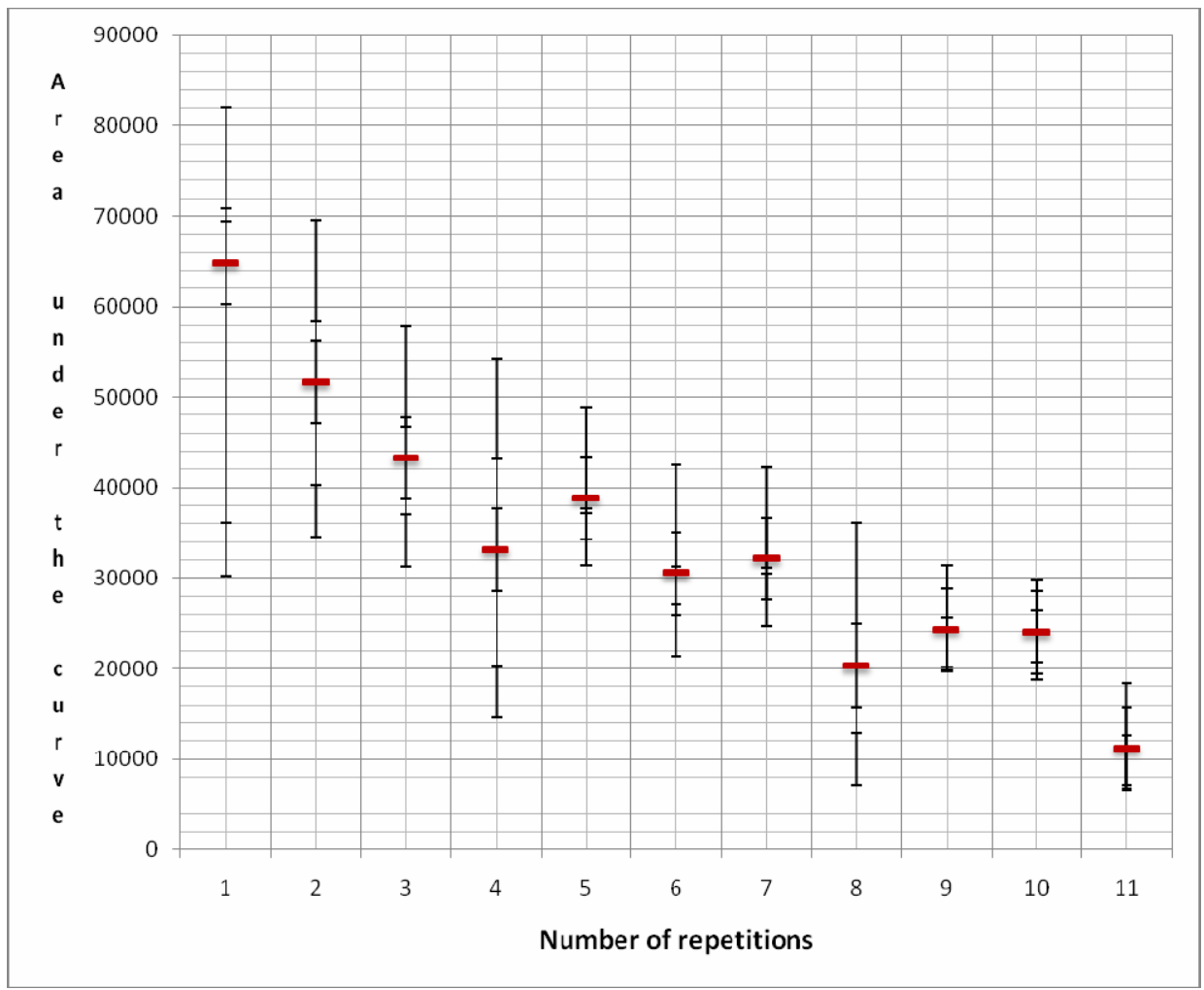

Fig. 11. "Work" developed during the transfer task

\section{Conclusions}

A new factor for the evaluation of laparoscopic training was developed. The method used $3 \mathrm{D}$ registers of each instrument during the execution of a specific task, using a mechatronic system. The adequate analysis of the three-dimensional signal during the execution of a task, demonstrates that after the training, the "work" invested in developing this task diminishes and is a factor that is measurable, and can be objectively reproduced for rating the skills of a laparoscopic surgeon.

\section{References}

Cotin S, Stylopoulos N, Ottensmeyer M, Neumann P, Rattner D, Dawson S. Metrics for Laparoscopic Skills Trainers: The Weakest Link! Lect Notes Comput Sc LNCS 2002;2488:35-43. 
Chmarra MK, Grimbergen CA, Dankelman J. Systems for tracking minimally invasive surgical instruments. Minim Invasiv Ther \& Allied Technol. 2007;16: 328-40.

Derossis AM, Fried GM, Abrahamowicz M, Sigman HH, Barkun JS, Meakins JL. Development of a model for training and evaluation of laparoscopic skills. Am J Surg 1998; 175: pp 482-487

Dankelman J, Chmarra MK, Verdaasdonk EGG, Stassen LPS, Grimbergen CA. Fundamental aspects of learning minimally invasive surgical skills. Minim Invasiv Ther \& Allied Technol. 2005; 14:247-56.

Martin JA, Regehr G, Reznick R, MacRae H, Murnaghan J, Hutchison C, et al.. Objective structured assessment of technical skills (OSATS) for surgical residents. Brit J Surg. 1997; 84:273-8.

Moore MJ, Bennett CL. The learning curve for laparoscopic cholecystectomy.Am J Surg. 1995; 170:550-9.

McClusky DAM, van Sickle K, Gallagher AG. Relationship between motion analysis, time, accuracy, and errors during performance of a laparoscopic suturing task on an augmented reality simulator. 12th International Congress of the European Association

Sutherland LM, Middleton PF, Anthony A, Hamdorf J, Cregan P, Scott D, et al.. Surgical simulation. A systematic review. Ann Surg. 2006; 243:291-300.

Schijven M, Jakimowicz J. Virtual reality surgical laparoscopic simulators. How to choose. Surg Endoscopy. 2003;17:1943-50.

Vassiliou MC, Feldman LS, Andrew CG, Bergman S, Leffondre K, Stanbridge D, et al. A global assessment tool for evaluation of intraoperative laparoscopic skills. Am J Surg. 2005;190:107-13.

Peters JH, Fried GM, Swanstrom LL, Soper NJ, Sillin LF, Schirmer B, et al. Development and validation of a comprehensive program of education and assessment of the basic fundamentals of laparoscopic surgery. Surgery 2004; 135: 21-27. 


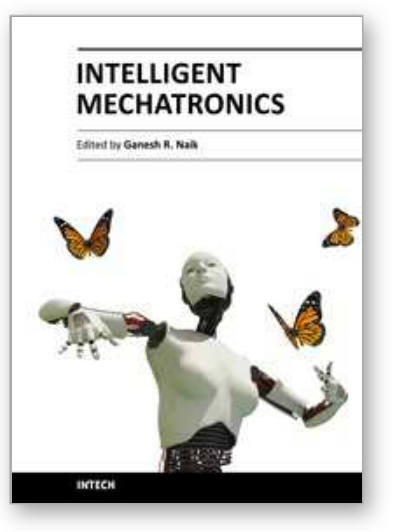

\author{
Intelligent Mechatronics \\ Edited by Prof. Ganesh Naik
}

ISBN 978-953-307-300-2

Hard cover, 248 pages

Publisher InTech

Published online 28, February, 2011

Published in print edition February, 2011

This book is intended for both mechanical and electronics engineers (researchers and graduate students) who wish to get some training in smart electronics devices embedded in mechanical systems. The book is partly a textbook and partly a monograph. It is a textbook as it provides a focused interdisciplinary experience for undergraduates that encompass important elements from traditional courses as well as contemporary developments in Mechtronics. It is simultaneously a monograph because it presents several new results and ideas and further developments and explanation of existing algorithms which are brought together and published in the book for the first time.

\title{
How to reference
}

In order to correctly reference this scholarly work, feel free to copy and paste the following:

Minor A., Lorias D., Ortiz Simon and Escamirosa F. (2011). Intelligent Mechatronic System for Automatically Evaluating the Training of the Laparoscopic Surgeon, Intelligent Mechatronics, Prof. Ganesh Naik (Ed.), ISBN: 978-953-307-300-2, InTech, Available from: http://www.intechopen.com/books/intelligentmechatronics/intelligent-mechatronic-system-for-automatically-evaluating-the-training-of-the-laparoscopicsurgeon

\section{INTECH}

open science | open minds

\section{InTech Europe}

University Campus STeP Ri

Slavka Krautzeka 83/A

51000 Rijeka, Croatia

Phone: +385 (51) 770447

Fax: +385 (51) 686166

www.intechopen.com

\section{InTech China}

Unit 405, Office Block, Hotel Equatorial Shanghai

No.65, Yan An Road (West), Shanghai, 200040, China

中国上海市延安西路65号上海国际贵都大饭店办公楼 405 单元

Phone: +86-21-62489820

Fax: +86-21-62489821 
(C) 2011 The Author(s). Licensee IntechOpen. This chapter is distributed under the terms of the Creative Commons Attribution-NonCommercialShareAlike-3.0 License, which permits use, distribution and reproduction for non-commercial purposes, provided the original is properly cited and derivative works building on this content are distributed under the same license. 